\title{
EBSD and Coupled EBSD/TEM Analysis of Zirconium Deformation Mechanisms
}

\author{
Rodney J. McCabe*, Gwenaelle Proust*, Amit Misra*, Ellen K. Cerreta*, Benjamin L. Henrie \\ *Materials Science and Technology Division, Los Alamos National Laboratory, Mail Stop G770, \\ Los Alamos, NM 87545
}

At temperatures between $76 \mathrm{~K}$ and room temperature, up to three twinning $(\{10 \overline{1} 1\}<\overline{1} 011>$, $\{11 \overline{2} 1\}<\overline{1} \overline{1} 26>,\{11 \overline{2} 2\}<\overline{1} \overline{1} 23>)$ and two slip mechanisms $(\{10 \overline{1} 0\}<1 \overline{2} 10>$ and $\{10 \overline{1} 1\}<\overline{1} \overline{1} 23>$ ) can contribute to the deformation of zirconium. A semi-automated, EBSD based method was used to quantitatively analyze the contribution of the three twinning modes to the deformation of Zirconium at $76 \mathrm{~K}$. The analysis involved characterizing twin boundaries as boundaries with a misorientation of $180 \pm 5^{\circ}$ about either the twinning $K_{I}$ plane or $\eta_{I}$ direction using TSL OIM Analysis 4.5 software. A program developed at Los Alamos was then used to classify portions of twinned grains as either parent or twins. The program classifies portions of twinned grains based on considerations of Schmid factors, relative sizes, aspect ratios, multiple twin variants, and/or twin generations [1]. The program has been updated to output an OIM Analysis readable file that can be used to visually verify and change the solutions where appropriate.

Measurements made with this method indicate a linear increase in primary $\{11 \overline{2} 2\}<\overline{1} \overline{1} 23>$ twinning fraction with strain to at least $20 \%$ strain for compression parallel to the primary c-axis texture component. A more significant finding is the important contribution of secondary twinning $(\{10 \overline{1} 1\}<\overline{1} 011>$ twins within primary $\{11 \overline{2} 2\}<\overline{1} \overline{1} 23>$ twins) with an exponential increase in secondary twin fraction with primary twin fraction. For measurements based only on evolving texture, this contribution creates erroneous results in estimates of twinning fractions and the illusion of early twin saturation. For compression perpendicular to the primary c-axis texture, primary $\{10 \overline{1} 1\}<\overline{1} 011>$ twinning increases linearly up to a strain of around $17 \%$ with very little secondary twinning to this point. Beyond $17 \%$ strain, secondary

$\{11 \overline{2} 2\}<\overline{1} \overline{1} 23>$ twinning begins to make a significant contribution to strain and results in a considerable decrease in the work hardening rate.

In order to investigate the role of slip mechanisms, particularly within deformation twins, a method coupling EBSD and TEM analysis was utilized. The method involves performing EBSD and TEM on the same TEM foil. Performing EBSD analysis prior to TEM allows for straightforward twin analysis and pre-selection of grains and/or twins of interest for dislocation or twin boundary analysis. Using this method, it was found that $\{10 \overline{1} 0\}<1 \overline{2} 10>$ slip within primary $\{11 \overline{2} 2\}<\overline{1} \overline{1} 23>$ twins and in grains that are oriented away from c-axis compression likely plays a considerable role in deformation during compression parallel to the primary c-axis texture.

1. B. L. Henrie and T. A. Mason, JOM, 56, (2004) 241 


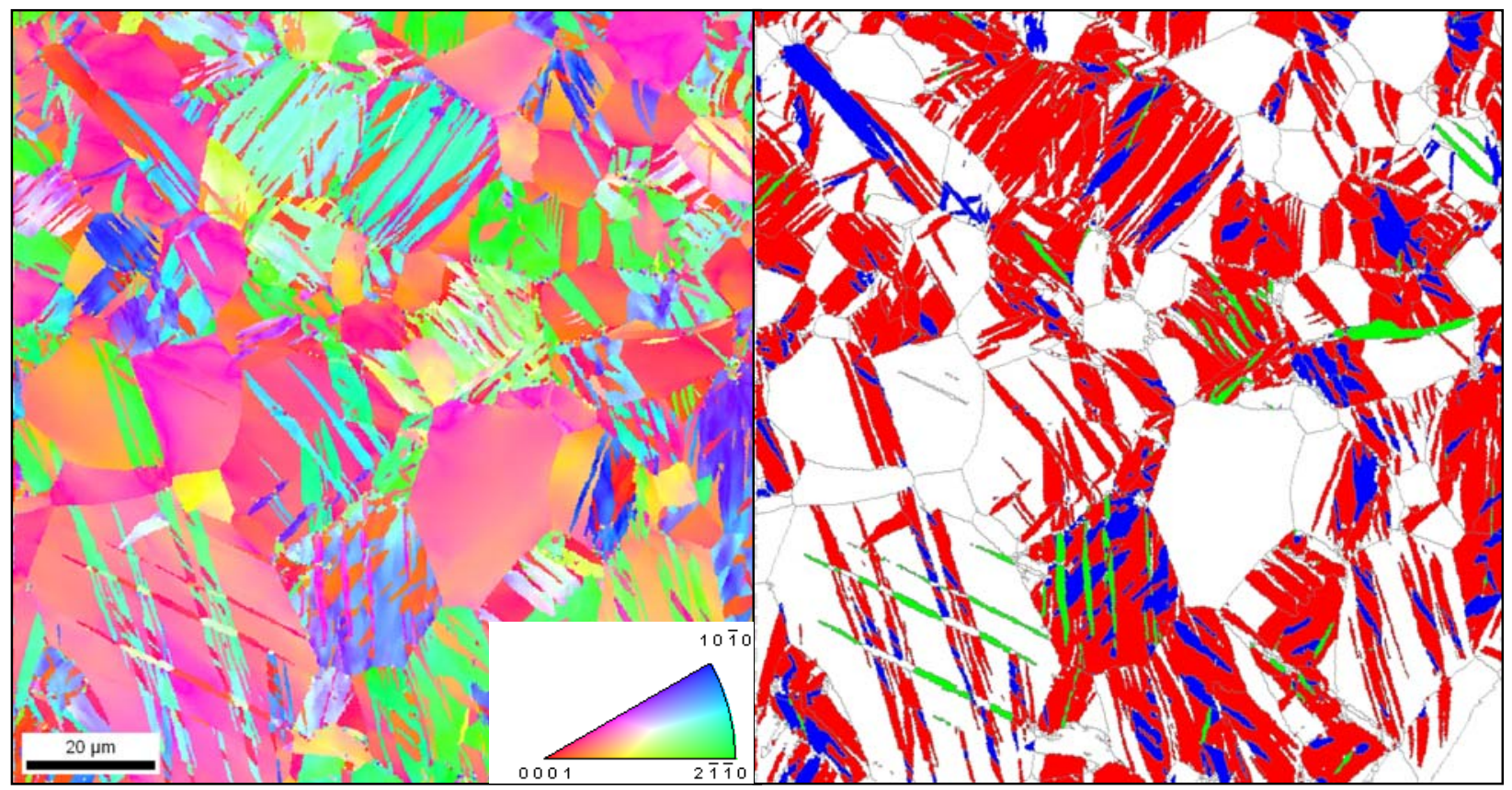

Figure 1. EBSD inverse pole figure (IPF) of a sample having undergone $10 \%$ compression parallel to the primary c-axis texture. The IPF displays the compression axis orientation as indicated by the standard hep orientation triangle. Prior to deformation, the compression axes of most grains were oriented within $15^{\circ}$ of the [0001] (c-axis) orientation. The right image shows the twinning structure. The $\{11 \overline{2} 2\}<\overline{1} \overline{1} 23>$ (red) twins are primary twins and most of the $\{10 \overline{1} 1\}<\overline{1} 011>$ (blue) and $\{11 \overline{2} 1\}<\overline{1} \overline{1} 26>$ (green) twins are secondary twins and are seen to be within primary $\{11 \overline{2} 2\}<\overline{1} \overline{1} 23>$ twins.
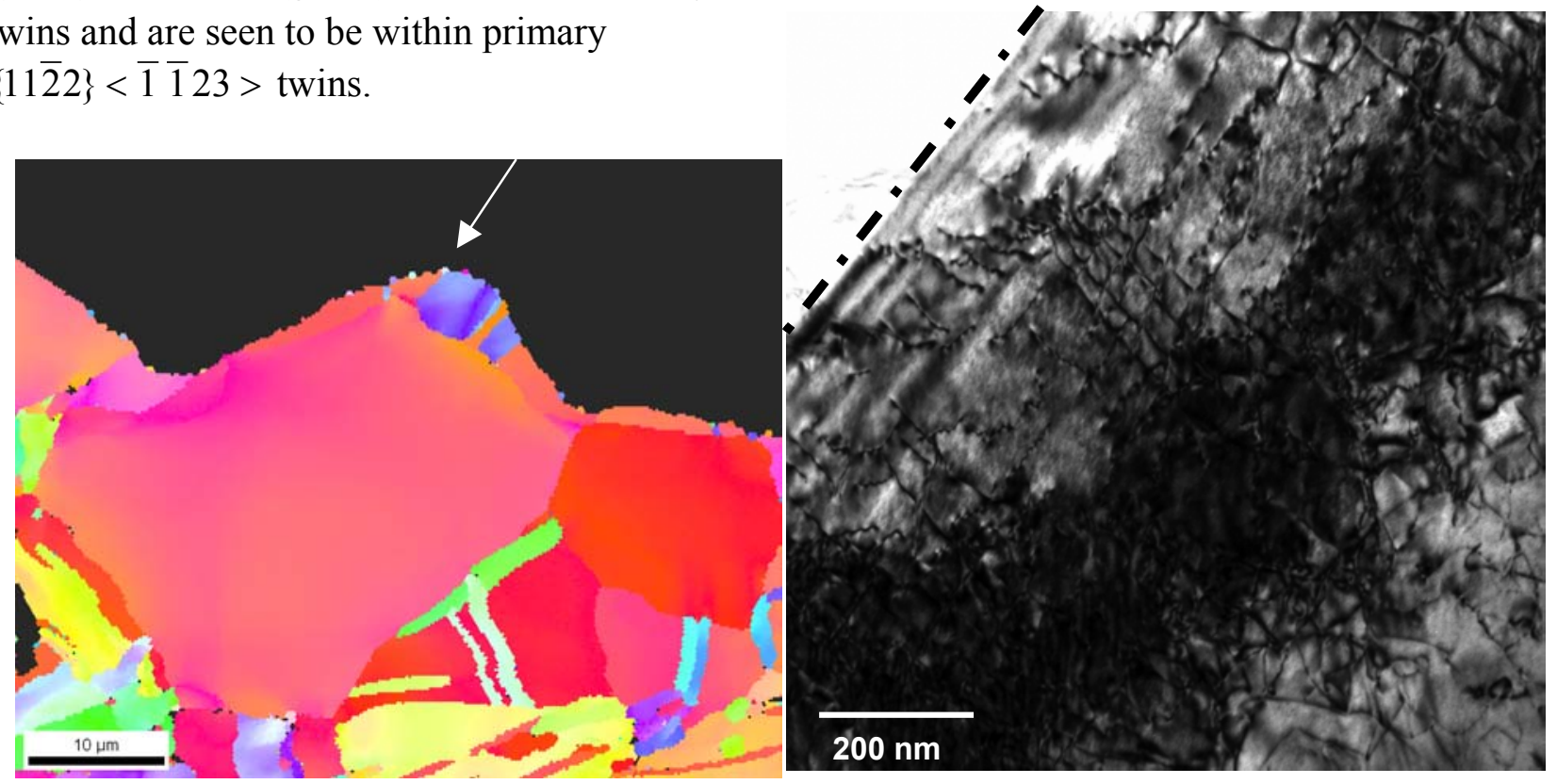

Figure 2. EBSD IPF of a region of a TEM foil where the orientation and compression condition are similar to the IPF in Figure 1. The blue region is a $\{11 \overline{2} 2\}<\overline{1} \overline{1} 23>$ twin and the arrow points to the boundary shown in the TEM image at the right. The twin is shown in the TEM image to contain a large number of $\{10 \overline{1} 0\}<1 \overline{2} 10>$ dislocations. Most of these dislocations are out of contrast for $\mathbf{g}=(0002)$ 